\title{
Ions Tune Interfacial Water Structure and Modulate Hydrophobic Interactions at Silica Surfaces
}

Aashish Tuladhar ${ }^{a, \dagger, t}$, Shalaka Dewan ${ }^{a, \dagger}$, Simone Pezzotti ${ }^{a, \#}$, Flavio Siro Brigiano, ${ }^{, \#}$, Fabrizio Creazzo $^{\#}$, Marie-Pierre Gaigeot*,\#, and Eric Borguet ${ }^{*, \dagger}$

†Department of Chemistry, Temple University, 1901 N. 13th St., Philadelphia, PA 19122, USA

† Physical \& Computational Sciences Directorate, Pacific Northwest National Laboratory, Richland, WA 99352

"LAMBE UMR8587, Université d'Evry val d'Essonne, CNRS, CEA, Université Paris-Saclay, 91025, Evry, France

${ }^{a}$ These authors contributed equally to this work.

Corresponding Authors

* Tel.: 33 1-69-47-01-40Ｅ-mail: mgaigeot@univ-evry.fr

* Tel.: 1-215-204-9696. E-mail: eborguet@temple.edu

\section{Supporting Information}




\section{S1 - Experimental setup.}

Optical Setup. To access the vibrational lifetimes of the interfacial O-H stretch, one-color IR pump - vSFG probe measurements were performed at the silica/water interface using the experimental setup described in previous studies. ${ }^{1-5}$ In brief, a one box Ti:Sapphire oscillator + regenerative amplifier (Coherent, LIBRA -F-1K-110-HE+) produces $5.0 \mathrm{~mJ}$ at $800 \mathrm{~nm}$ with a pulse duration of $120 \mathrm{fs}$ at a repetition rate of $1 \mathrm{kHz}$ (Figure $\mathrm{S} 1) .90 \%$ of the output $(4.5 \mathrm{~mJ})$ was used to pump a commercial OPA (Coherent, TOPAS-Prime HE) to produce $1.3 \mathrm{~W}$ of signal and idler. The DFG/OPA combination was tuned to generate IR pulses, centered at $3200 \mathrm{~cm}^{-1}$ with a typical pulse energy of $20 \mu \mathrm{J} @ 3 \mu \mathrm{m}\left(\sim 200 \mathrm{~cm}^{-1} \mathrm{FWHM}\right)$, which were then split in a $70 \%-30 \%$ ratio to be used as IR pump and IR probe, respectively. The remaining $5 \%$ of the regenerative amplifier output, spectrally narrowed to a FWHM of $\sim 2.5 \mathrm{~nm}\left(38 \mathrm{~cm}^{-1}\right)$ using a narrow bandpass filter, was used as the visible light for vSFG measurements. The infrared probe and visible beams that generate the vSFG probe have incident angles of $65^{\circ}$ and $70^{\circ}$, respectively, with respect to the surface normal, while the IR pump was incident at an angle of $75^{\circ}$ with respect to the surface normal (Figure S2). The incident angles were selected based on the best Fresnel factor enhancement under total internal reflection, similar to previous studies..$^{2-3,6-7}$ The focal spot diameters of the IR pump, IR probe, and VIS probe were $150 \mu \mathrm{m}, 150 \mu \mathrm{m}$ and $200 \mu \mathrm{m}$, respectively. All incident beams were $\mathrm{p}$ polarized and the vSFG signal was collected in $\mathrm{p}$ polarization.

Sample Preparation. IR transmitting amorphous fused $\mathrm{SiO}_{2}$ triangular roof prisms $(15 \times 13 \times 13$ x $15 \mathrm{~mm}$ ) with the $15 \times 15 \mathrm{~mm}$ square face (opposite of the roof) were purchased from Team Photon Inc. (San Diego, CA). Before the experiment, the $\mathrm{SiO}_{2}$ triangular prism was first cleaned with freshly prepared "piranha" solution (1 volume concentration $\mathrm{H}_{2} \mathrm{O}_{2}: 3$ volume concentration 
$\mathrm{H}_{2} \mathrm{SO}_{4}$ ) for $\sim 30$ minutes in a Teflon holder. (CAUTION: "piranha" is a very reactive mixture and must be handled with great care by using protective equipment including appropriate gloves, googles, and lab coat). This was followed with copious amount of rinsing with deionized water $(>18.2 \mathrm{M} \Omega \cdot \mathrm{cm}$ resistivity, Thermoscientific Barnstead Easypure II purification system with a UV lamp) and then the prism was dried by filtered compressed $\mathrm{N}_{2}$ gas. The $\mathrm{SiO}_{2}$ prism was then assembled onto the sample holder and exposed to neat water immediately and allowed to equilibrate in ambient at room temperature before experiments.

The silica/water interface was achieved by pressing the silica prism against a volume of water in a home-built sample cell (Fig. S2). The design of the sample cell allowed for an easy exchange of the aqueous media without moving the prism. This provision prevents any change in the position of the interface.

All solutions were prepared using deionized water, the $\mathrm{pH}$ of which was $\sim 6$ after equilibration with the laboratory ambient. Solutions of $\mathrm{pH} 2$ and 12 were made by dropwise adding small amounts of concentrated $\mathrm{HCl}(\sim 12.0 \mathrm{M}$, Fisher Scientific, analytical grade) and $6 \mathrm{M} \mathrm{NaOH}$ (Fluke Analytical, analytical grade), respectively, till the $\mathrm{pH}$ of the solution reached the desired level as measured by a pH meter (Oakton). Salt solutions of $0.1 \mathrm{M}$ and $0.5 \mathrm{M}$ were prepared using $\mathrm{NaCl}$ (Fisher Scientific, $>99.8 \%$ ), which was baked overnight at $500{ }^{\circ} \mathrm{C}$ to remove possible organic impurities. 


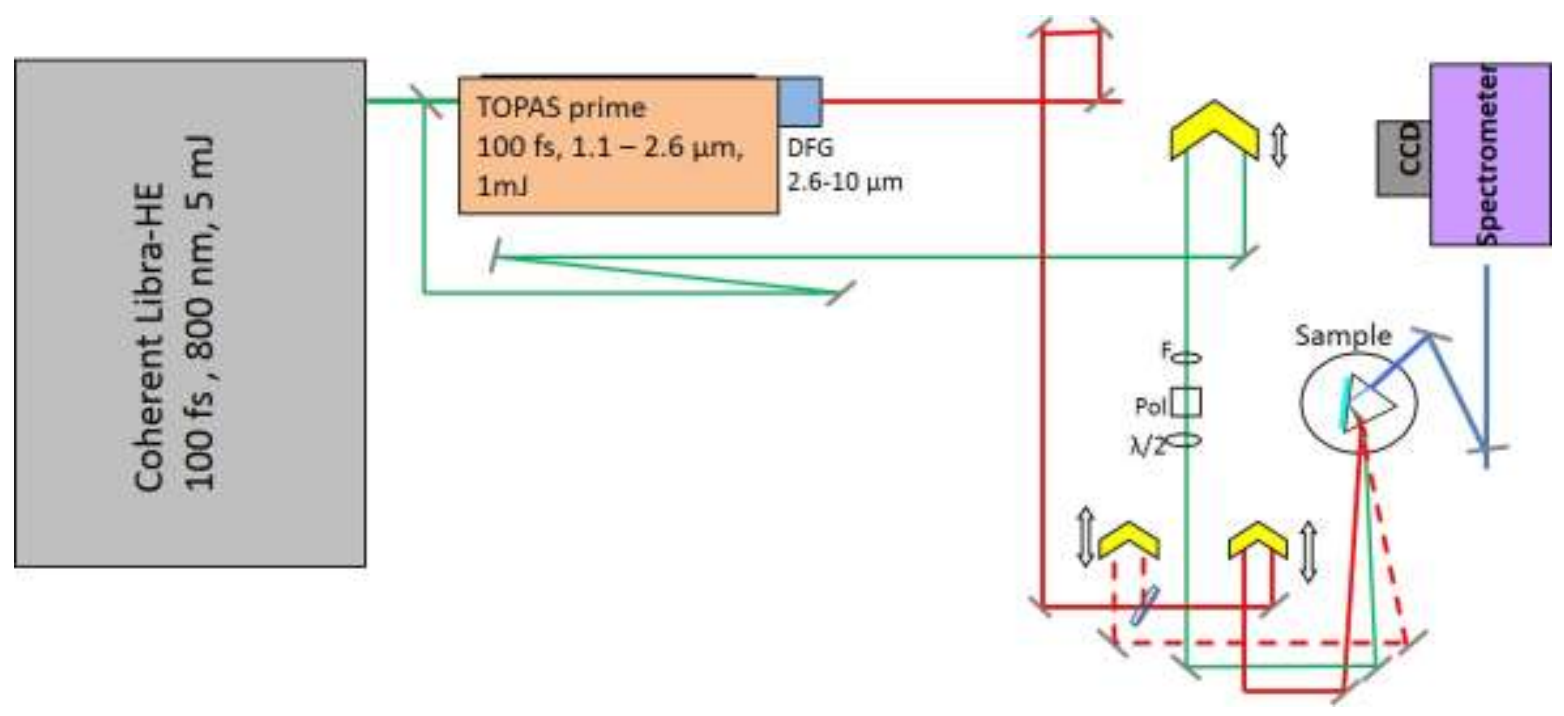

Figure S1: Schematic of the laser system and the experimental set up for frequency SFG measurements with the Libra-HE laser system, used to perform one-color TR-vSFG measurements

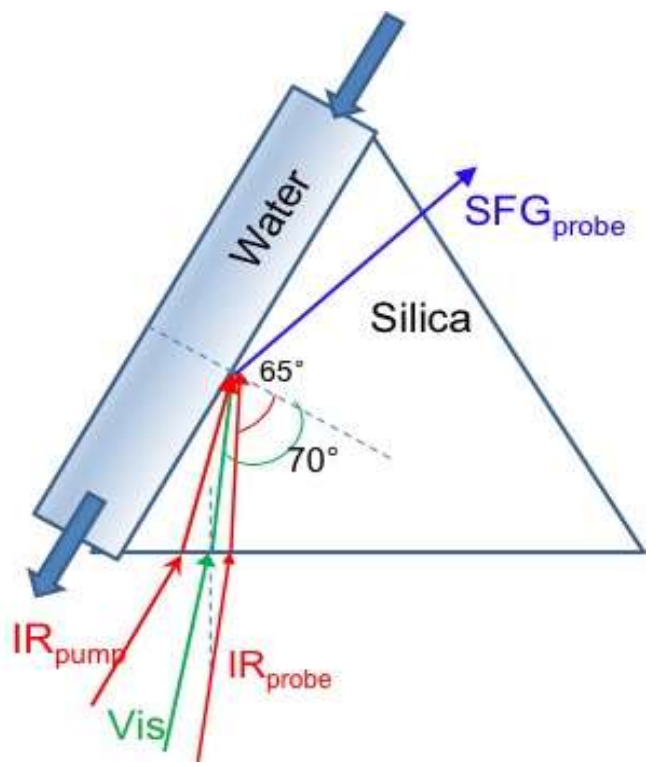

Figure S2: Diagram of the TR-vSFG measurements performed under total internal reflection conditions at the silica/water interface 
Experimental Details. Previous TR-vSFG studies have shown that at the silica/ $\mathrm{H}_{2} \mathrm{O}$ interface, the vibrational dynamics do not exhibit any frequency dependence ${ }^{2,8}$. In other words, for one-color TR-vSFG, we expect similar results for strongly and weakly hydrogen bonded water, i.e., the same dynamics for IR frequencies centered at $3200 \mathrm{~cm}^{-1}$ or $3400 \mathrm{~cm}^{-1}$. Owing to better signal to noise of the vSFG spectra obtained at $3200 \mathrm{~cm}^{-1}$ region, all the results discussed here are pumped and probed at $3200 \mathrm{~cm}^{-1}$ region of the $\mathrm{O}-\mathrm{H}$ stretch, which corresponds to the strongly hydrogen bonded water species. Additionally, it is important to mention that usually the bleach signal can be accompanied by a hot band response. However, due to anharmonicity, the hot band of O-H stretch is $200-400 \mathrm{~cm}^{-1}$ red-shifted from the fundamental $\mathrm{O}-\mathrm{H}$ stretch and therefore, outside the window of our probe vSFG (3100 to $\left.3300 \mathrm{~cm}^{-1}\right)$. The tail of the hot band might affect the bleach dynamics, but we expect its contribution to be insignificant on the $\mathrm{T}_{1}$ lifetime, since we are primarily probing the population at the $3200 \mathrm{~cm}^{-1}$ region (ground state $\mathrm{OH}$ stretch).

The vibrational relaxation of interfacial water at a silica surface is determined by the recovery of the bleach in the vSFG intensity as a function of the delay between the IR pump and the vSFG probe. The kinetics of vibrational relaxation is modeled using a four level system (Figure S3), already extensively used in previous work to describe the dynamics of bulk water ${ }^{9-11}$ as well as water at mineral oxide surfaces ${ }^{1-5,8,12}$.

\section{S2 - Model used to describe vibrational dynamics of O-H in water:}

In this model, the vibrational energy of the excited $v=1$ state is first transferred to an intermediate state $v^{*}$ state, via inter- and intramolecular energy transfer to the overtone of the bend, or via resonant energy transfer to the $\mathrm{O}-\mathrm{H}$ stretches of other water molecules that may be are coupled via hydrogen bonds ${ }^{2}$. This occurs with a relaxation time $\left(\mathrm{T}_{1}\right)$, which corresponds to the vibrational lifetime of the first excited state $(v=1)$ of the $\mathrm{O}-\mathrm{H}$ oscillator. The relaxation from the intermediate 
$v^{*}$ state to a hot ground state $v_{0}{ }^{*}$ occurs via a thermalization process with a time constant of $T_{\text {th. }}$. The thermalization step involves the statistical distribution of the remaining vibrational energy to all possible modes, including librations and the hydrogen bond reorganization ${ }^{13-14}$.

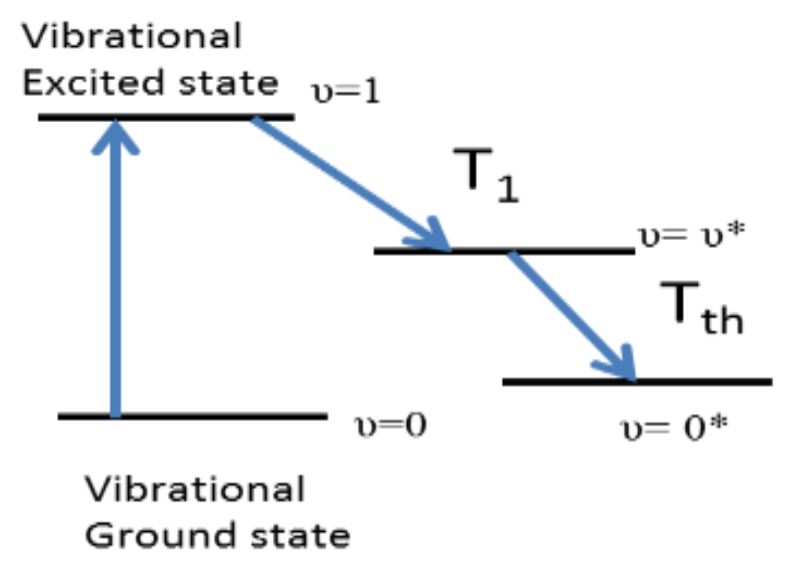

Figure S3: Four level model used to describe vibrational relaxation of water in the bulk and at interfaces.

The time-resolved vSFG data can be fit to the following equation, which describes the kinetics of vibrational relaxation:

$$
I_{S F G}=\operatorname{IRF}(\mathrm{t}) *\left[N_{0}(t)-N_{1}(t)+C_{2} N_{2}(t)+C_{3} N_{3}(t)\right]^{2}
$$

where $\mathrm{N}_{0}, \mathrm{~N}_{1}, \mathrm{~N}_{2}$, and $\mathrm{N}_{3}$ are the populations of the $v=0, v=1, v^{*}$, and $v^{*}=0$ states, respectively, and are determined by the solution to the system of coupled differential equations that describe the rates of population change for the four levels. $\mathrm{C}_{2}$ and $\mathrm{C}_{3}$ are factors related to the strengths of the vSFG signal from the intermediate and final levels, and $\operatorname{IRF}(\mathrm{t})$ represents the Gaussian profile of the instrument response function (IRF). 
Since our experiments were not sensitive to the thermalization effect, the $T_{\text {th }}$ value was held constant to a previously reported ${ }^{2-3}$ value of 700 fs while fitting the data. The insensitivity of our data towards the thermalization effect probably stems from the pump IR and probe IR being identical. If we were to pump the strongly hydrogen bonded region and probe the weakly hydrogen bonded region, the thermalization timescale can be more accurately measured. However, this is not the scope of our investigation.

\section{S3 - Calculated surface electric potential}

The silica surface charge $\left(\sigma_{0}\right)$ was calculated at each $\mathrm{pH}$ and ionic strength using Equation (S2):

$$
\sigma_{0}=\sigma_{\max } \times\left(\theta>\operatorname{SiO}_{\text {total }}\right)
$$

where $\sigma_{\max }\left(\sim 0.72 \mathrm{C} / \mathrm{m}^{2}\right)$ is the maximum surface charge generated by deprotonation of all the silanol groups $\left(4.5 \mathrm{SiOH} / \mathrm{nm}^{2}\right)$ on the silica surface and $\left(\theta>\mathrm{SiO}_{\text {tot }}\right)$ is the total number of deprotonated sites $\left(\mathrm{SiO}^{-}\right.$and $\left.\mathrm{SiO}^{-} \mathrm{Na}^{+}\right)$obtained from Dove's model ${ }^{15}$. The surface potential $\left(\phi_{0}\right)$ was estimated from the Gouy-Chapman model as ${ }^{16-17}$ :

$$
\emptyset_{0}=\frac{2 k T}{z e} \sinh ^{-1}\left(\frac{\sigma_{0}}{\sqrt{8 k T \varepsilon_{0} \epsilon N_{A} I}}\right)
$$

where $k, N_{A}, T, I$, and $z$ are Boltzmann's constant, Avogadro's number, the absolute temperature, the ionic strength of the solution, and the electrolyte valence, respectively, and $\varepsilon_{0}$ and $\epsilon$ are the permittivity of free space and dielectric constant of the electrolyte. The calculated surface potential (Figure S4) using Equation (S3) at the silica surface with background electrolyte of $0.5 \mathrm{M} \mathrm{NaCl}$ is in good agreement with the measured surface potential reported by Eisenthal et al. ${ }^{18}$ 


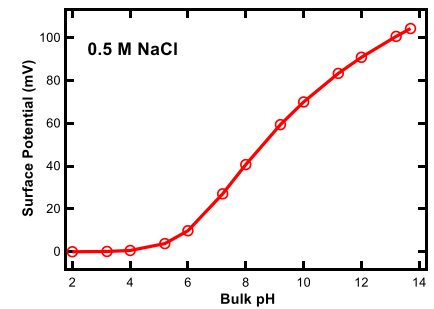

Figure S4: Calculated surface potential using the Gouy-Chapman model (Equation S3) as a function of bulk $\mathrm{pH}$ at $0.5 \mathrm{M} \mathrm{Na}^{+}$ionic strength. It is in good agreement with the measured surface potential by Eisenthal et al. ${ }^{18}$

The decay of the potential as a function of distance from the silica surface can be estimated by solving the Poisson-Boltzmann equation as ${ }^{16-17}$ :

$$
\varnothing(x)=\frac{4 k T}{z e} \tanh ^{-1}\left\{\tanh \left(\frac{z e \emptyset_{0}}{4 k T}\right) e^{-\kappa x}\right\}
$$

where $\kappa$ is the inverse of the Debye length and can be estimated as ${ }^{17}$ :

$$
\kappa=\sqrt{\frac{2 z^{2} e^{2} N_{A} I}{\varepsilon_{0} \varepsilon k T}}
$$

The drop of the surface potential at $\mathrm{pH} 2,6$, and 12 in the presence of $0,0.1 \mathrm{M}$, and $0.5 \mathrm{M} \mathrm{NaCl}$ is shown in Figure S5. 

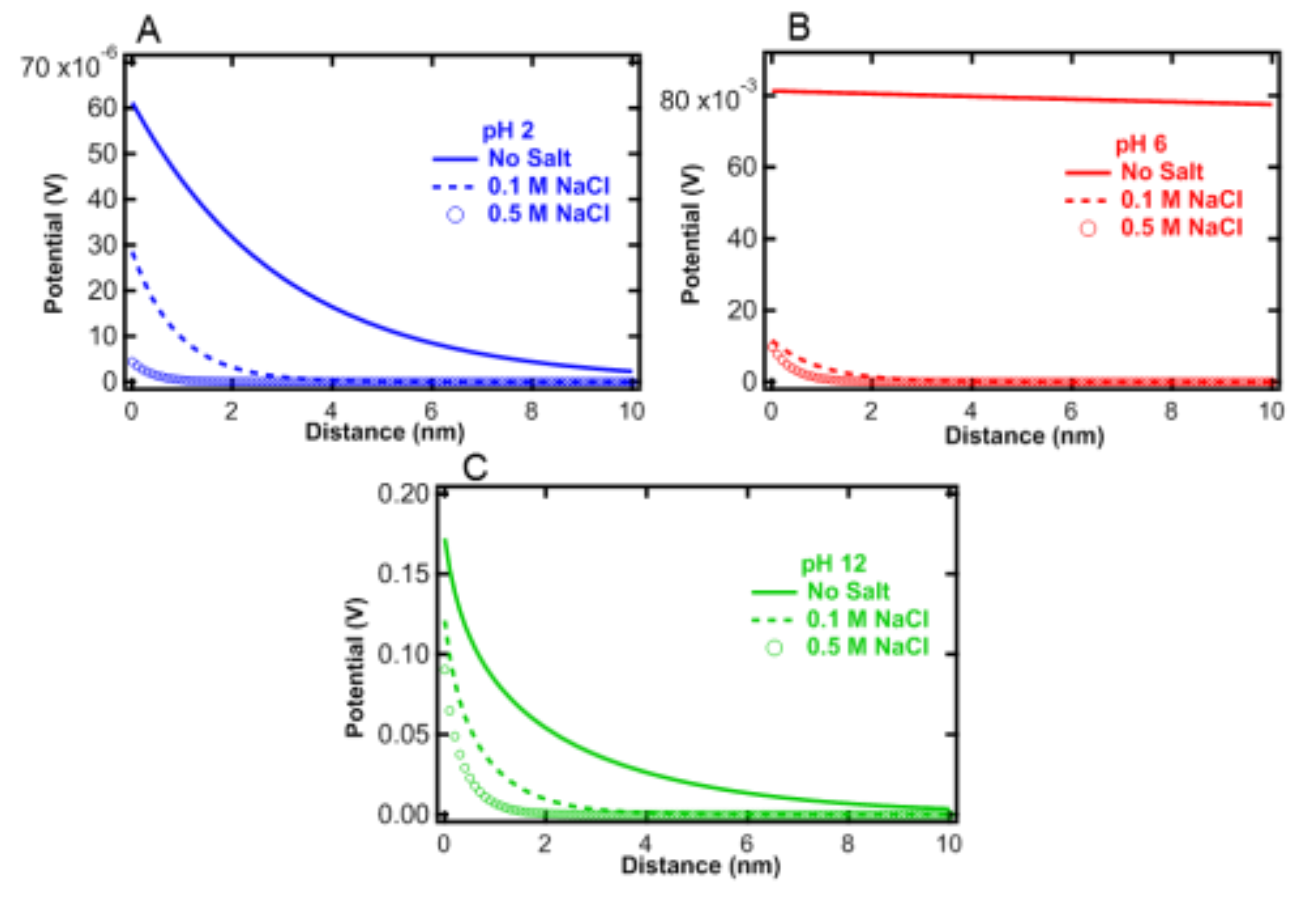

Figure S5: Calculated drop in surface potential as a function of distance using the Poisson-Boltzmann model (Equation S4) at bulk pHs (A) 2, (B) 6, and (C) 12 at varying ionic strength of $0 \mathrm{M}, 0.1 \mathrm{M}$, and $0.5 \mathrm{M} \mathrm{NaCl}$

\section{S4 - SS-vSFG signal counts at pH 2 conditions}

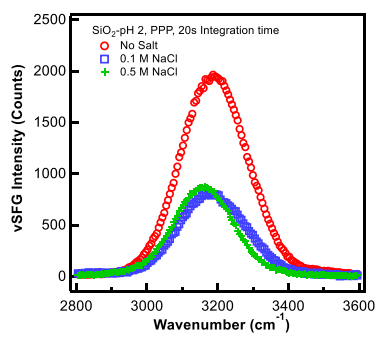

Figure S6: Non-normalized steady-state vSFG signal counts at $\mathrm{SiO}_{2}-\mathrm{pH} 2$ conditions in the presence of bulk $\mathrm{NaCl}$ concentrations of $0 \mathrm{M}$ (red circle), $0.1 \mathrm{M}$ (blue square), and $0.1 \mathrm{M}$ (green plus). 


\section{S5 - AIMD/DFT-MD molecular dynamics simulations.}

Density Functional Theory-based Molecular Dynamics simulations (DFT-MD or AIMD for Ab Initio MD) have been carried out on amorphous silica-liquid water interfaces. See a snapshot in Figure S7. The amorphous silica model is taken from Ugliengo et $a l^{19}$, with a $4.5 \mathrm{SiOH} / \mathrm{nm}^{2}$ silanol coverage of the surface in contact with the air (i.e. eight $\mathrm{SiOH}$ groups in our simulation box). Once put in contact with water, the surface is found stable along the simulation length ( $30 \mathrm{ps})$. The 4.5 $\mathrm{SiOH} / \mathrm{nm}^{2}$ silanol coverage is typical of hydrophilic silica surfaces ${ }^{19}$ and typical of the experimental conditions close to the PZC potential of zero charge $(\mathrm{pH} 2-4)^{15}$. The PZC condition also corresponds to the isoelectric point for aqueous amorphous silica. This is shown in section S6 where we define water layers at increasing distance from the surface and water structural properties within each layer are characterized. Our results clearly show that at the amorphous silica/water interface at PZC condition the interfacial layer is solely composed by the BIL, which is directly followed by bulk water. Since no DL is observed at this interface, we can conclude that PZC condition indeed corresponds to the isoelectric point.

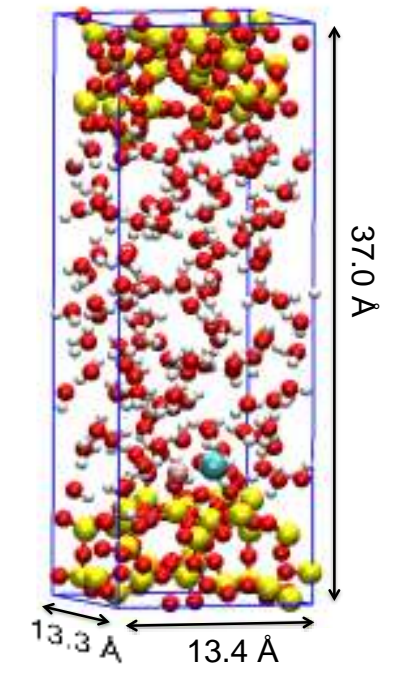

Figure S7. Snapshot of the DFT-MD simulation box, with amorphous silica, water and the $\mathrm{KCl}$ ion pair (Contact Ion-Pair in an inner-sphere adsorption). 
The DFT-MD simulations were conducted with the CP2K software package ${ }^{20}$, consisting in Born Oppenheimer MD, BLYP ${ }^{21-22}$ electronic representation including Grimme D2 correction for dispersion $^{23}$, GTH pesudopotentials ${ }^{24}$ and a combined plane waves (400 Ry energy cut-off) and SR-DZVP-MOLOPT Gaussian basis set for all atoms. The dimensions of the simulation box are 13.386 $\AA$ X 13.286 $\AA$ X $37.0 \AA$, the box is periodically repeated in all directions of space. The silica slab is $15 \AA$ thick, composed of 204 atoms, while the liquid water is modeled with 120 molecules in the box, providing the required $1 \mathrm{~g} / \mathrm{cm}^{3}$ liquid density. The amorphous silica slab was re-optimized in the gas phase at the level of theory adopted for the dynamics, then put in contact with bulk water. After an equilibration run of $10 \mathrm{ps}$ (5 ps with possible rescaling of velocities plus $5 \mathrm{ps}$ in the pure NVE ensemble) the dynamics was run in the NVE ensemble for 20 ps with a time step of $0.4 \mathrm{fs}$, where data are collected and analyzed.

Nine DFT-MD simulations of the amorphous silica-water interface in presence of salt were conducted on the same system, including one $\mathrm{NaCl}$ (note $\mathrm{KCl}$ is replaced by $\mathrm{NaCl}$ in one of the nine simulations) ion-pair in contact with the neat amorphous silica surface. The simulations setup is the same as for the case of the neat silica-water interface. The only exception is the plane wave cutoff used for the simulation with $\mathrm{NaCl}$, which has been enlarged to $1000 \mathrm{Ry}$ in order to properly sample the $\mathrm{Na}^{+}$diffusion. All simulations consists of a 5 ps equilibration run ( $2.5 \mathrm{ps}$ with possible rescaling of velocities plus $2.5 \mathrm{ps}$ in the pure NVE ensemble) and a subsequent dynamics in the NVE ensemble, with a time step of $0.4 \mathrm{fs}$ and simulations lengths in the $15 \mathrm{ps}-25 \mathrm{ps}$ range for all trajectories, for a total of $150 \mathrm{ps.}$ In these additional simulations, the initial position of the ion pair is randomly varied in the box, to check the dependence of the MD results on the initial configuration, as detailed in section S8 (see also Table S1). 
As the silica surface is modelled at the isoelectric point at PZC conditions, there is no surface electrostatic driving force to favor cations over anions (and vice versa) to approach closer to the silica surface when adding electrolytes at the $\mathrm{SiO}_{2} /$ water interface. This is demonstrated in section S6 by showing that there is not a DL layer at the neat silica-water interface, and it is confirmed by the surface potential estimations (Figure S5). Three generic scenarios can hence be envisaged in the relative position of $\mathrm{K}^{+}$and $\mathrm{Cl}^{-}$at $\mathrm{SiO}_{2}$ /water interfaces: 1) the two ions are simultaneously located in the BIL and interact with the silica surface, 2) $\mathrm{K}^{+}\left(\right.$resp. $\left.\mathrm{Cl}^{-}\right)$is in the $\mathrm{BIL}$ while $\mathrm{Cl}^{-}($resp. $\mathrm{K}^{+}$) is in the DL, 3) both ions are in liquid water (DL or Bulk). Scenario-1 is compatible with the PZC isoelectric conditions at the silica/water interface, while scenario-2 would induce a nonnegligible surface potential (incompatible with the estimated close to zero electrostatic potential at the electrolytic interface, see S3-2) and scenario-3 is of no importance for the interfacial properties.

We have therefore chosen to include $\mathrm{KCl} / \mathrm{NaCl}$ in the BIL-water (i.e. at the direct interface with the surface, see BIL/DL definitions in S5) by adding one ion pair in the simulation box at the $\mathrm{SiO}_{2} /$ water interface.

Both ions remain in the BIL over the total simulation time (see details in section S8), without significant vertical diffusion of the ionic species over this time-scale. Note that we have shown in refs $^{25-26}$ that $\mathrm{NaCl}$ and $\mathrm{KCl}$ behave identically at silica-water interfaces. We refer to Figure $\mathrm{S} 7$ for a snapshot of the system extracted from the simulation. The $\mathrm{KCl} / \mathrm{NaCl}$ ion-pair is furthermore shown (see main text) to preferentially adsorb in an inner-sphere mode. 


\section{S6 - Characterization of BIL and DL water layers in molecular dynamics simulations.}

A critical issue in calculating and interpreting vSFG nonlinear spectra (and associated $\mathrm{T}_{1}$ relaxation times) is a clear and unambiguous definition of the water thickness contributing to the total signal, i.e., the extent of the region where the water is non-centrosymmetric. The pioneering work of Tian

et $a l .{ }^{27}$ has provided a framework comprised of the two universal water layers, named BIL (Binding Interfacial Layer) and DL (Diffuse Layer), solely responsible for vSFG activity at any interface (only the BIL contributes to the vSFG spectra at neutral/isoelectric interfaces). In refs ${ }^{28-}$ ${ }^{30}$, we have developed, applied and validated a theoretical protocol to unambiguously define these layers from (DFT)-MD simulations on the basis of water structural properties only. Our method uses three structural descriptors: (1) The water density profile as a function of the distance (z) from the surface, which provides the number of water layers and their z-boundaries; (2) The average number of hydrogen bonds formed by the water molecules within the layers found in 1); (3) 3Dplots of the distribution of water-water H-Bond lengths and H-Bond angles (defined with respect to the normal to the surface) that provide the general structural organization of water within the layers defined in 1 .

The layers where water has all three descriptors identical to bulk liquid water are termed 'liquid water'. This is further verified by checking that the associated vSFG spectrum is equal to zero, as it should indeed be for centrosymmetric liquid water. The layers where all descriptors are identical to bulk liquid water except for the orientation of the water-water H-Bonds along one given direction of space are associated to the DL (Diffuse Layer). This DL is indeed bulk water reoriented by the surface charge potential/field, and therefore is not centrosymmetric anymore. The DL can hence be seen as bulk water under the influence of an external field. The layers where all descriptors are different from bulk liquid water are called the BIL. From our previous works ${ }^{28-}$ 
29,31 , the BIL has been systematically found to be composed of one monolayer of water, within $\sim 3$

A thickness. The present work makes no exception to this result.
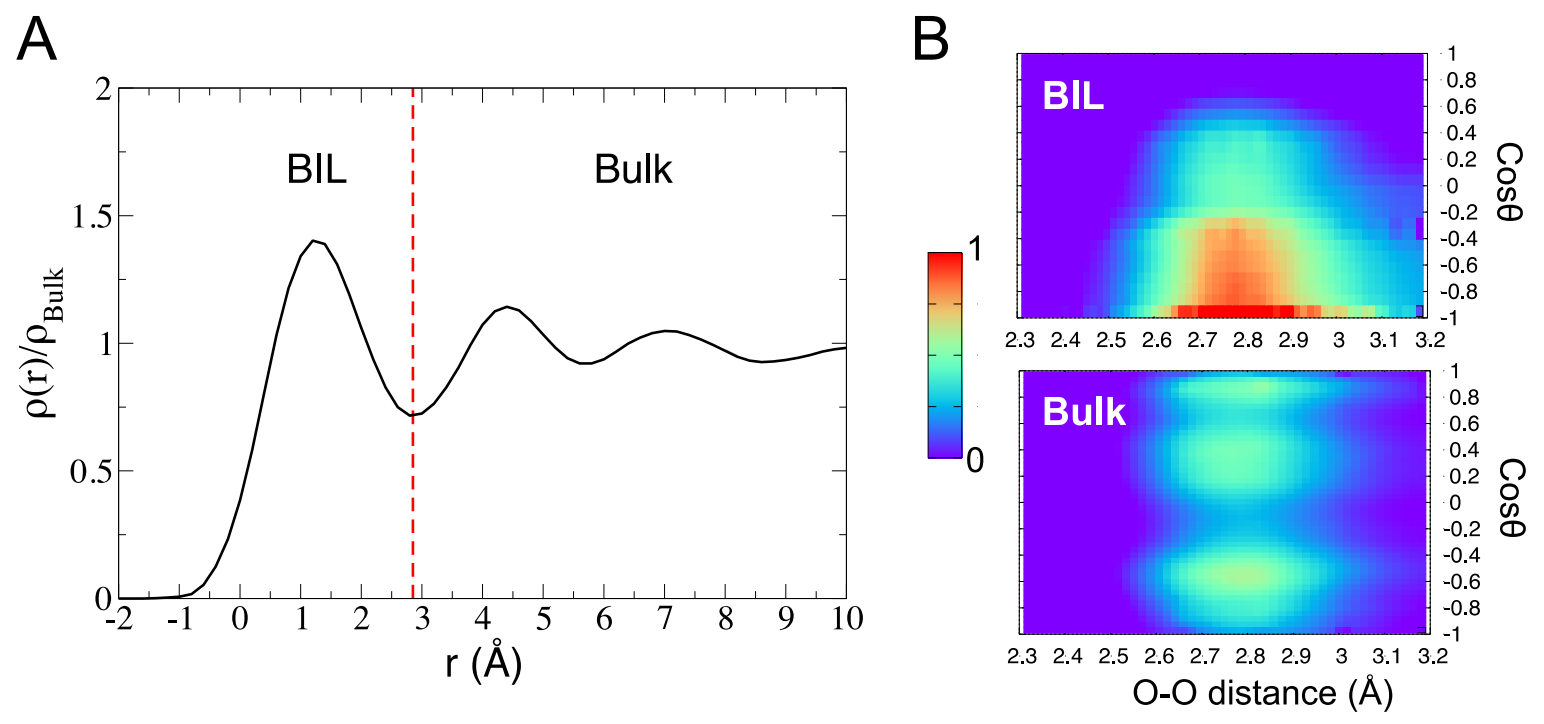

Figure S8. Two of the three descriptors used for characterizing and extracting water layers at neutral and charged interfaces. The third descriptor is the average number of $\mathrm{HBs} /$ molecule in each layer and is discussed in the text.

A: Time averaged water density profile (normalized with respect to bulk water density) as a function of the distance from the instantaneous surface for the neat silica-water interface. The boundary between the BIL and the DL is marked with a red dashed line.

B: 3D-plots of the HB patterns formed between the water molecules in the BIL and Bulk layers at the neat silica-water interface. The $\mathrm{x}$-axis reports the $\mathrm{O}-\mathrm{O}$ distance between two $\mathrm{H}$-Bonded water molecules and the $\mathrm{y}$-axis provides the angle (cosine values) between the $\mathrm{O}-\mathrm{O}$ vector (from donor to acceptor) and the normal to the surface (oriented from liquid to solid phase). The color represents the probability to find one $\mathrm{O}-\mathrm{H}$ group in the BIL/Bulk layer forming one HB with a given distance and orientation.

The same methodology has been applied here to the simulated amorphous silica-water interfaces, neat (without electrolytes) and in presence one $\mathrm{KCl}$ ion pair (roughly corresponding to $1 \mathrm{M}$ concentration in the simulations). The density profile and 3D-plots for the neat silica-water interface are presented in Figure S8, in order to show the layer characterization and definition. The BIL layer corresponds to the first peak in the density profile. It has a higher density than bulk 
water. In this layer, water molecules form on average $3.0 \mathrm{HBs} /$ molecule (including water-solid and water-water HBs), less than the value of $3.4 \mathrm{HBs} /$ molecule obtained from a reference simulation of bulk water (with the same computational setup) ${ }^{29}$. In addition, water molecules in this first layer are not in an isotropic environment, as shown in the 3D-plot (Figure S8 B - BIL), where one can see an inhomogeneous distribution in cosine values (in contrast with the homogeneous distribution in bulk water), as water molecules preferentially form water-water HBs with water located in the subsequent (bulk) layer (thus the red region at negative cosine values). Water in the BIL thus differs from bulk water for all the investigated structural properties. On the contrary, after this first layer, water molecules have an average coordination of 3.4 (equal to the value obtained for bulk water) and spatial orientation isotropy is recovered (homogeneous distribution in cosine values as shown in the 3D-plot). Bulk water is thus recovered after one water monolayer only at the investigated neutral silica-water interface.

In Figure S9 we present a schematic representation of the water layers at the neat (A panel) and 1M-electrolytic (B panel) amorphous silica/water interfaces characterized in the present work by DFT-MD simulations. These schemes are for PZC conditions. Figure 2 in the main text provides the three schemes at $\mathrm{pH}=2$ (PZC), 6, 12 conditions. See text above (and main text) for the justification of why both cations and anions are simultaneously located in the BIL water layer in our simulation at $\mathrm{PZC}(\mathrm{pH}=2)$ conditions (surface at the isoelectric point). 

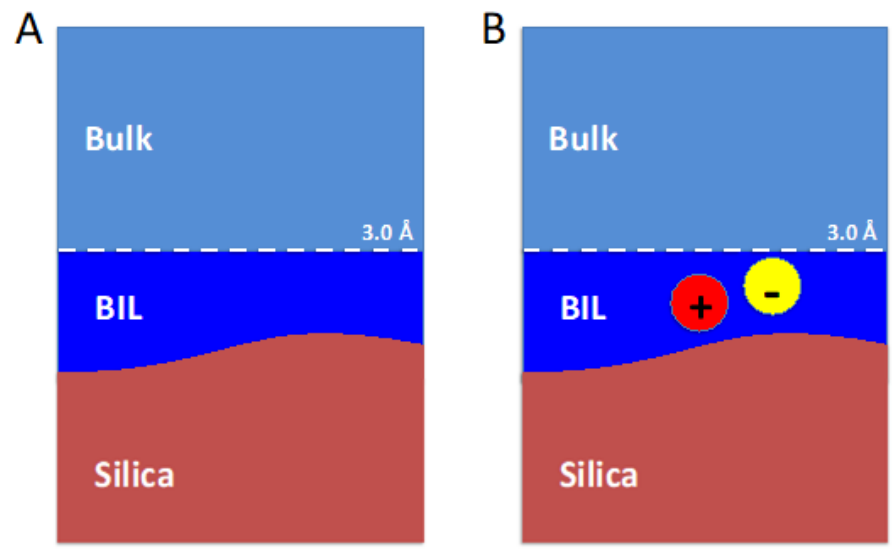

Figure S9. $\mathrm{pH}=2$ (PZC conditions), schematic representations of the water layers (BIL and Bulk) identified for the neat (A) and electrolytic (B) amorphous silica/water interfaces investigated in the two DFT-MD simulations performed in this work. The $3 \AA$ thickness of the BIL interfacial region is highlighted in dark blue in the figure. For the electrolytic interface (B) the location of the cation and anion in the BIL is also highlighted. The SI and the main text provide explanations of why the two ions of the electrolyte are simultaneously located in the BIL water layer at PZC conditions.

\section{S7 - Coordination of water mapped in the BIL.}

The time averaged spatial distribution of the coordination number of water in the BIL shown in Figures $3 \mathrm{~A}$ and $3 \mathrm{~B}$ in the main text is obtained by the following procedure. Two water molecules are considered hydrogen bonded, and therefore coordinated, if the O-O distance is less than $3.2 \AA$ and if the $\mathrm{O}(-\mathrm{H})-\mathrm{O}$ angle is in the range of $140-220^{\circ}$. This is the standard $\mathrm{HB}$ criterion $^{30}$. The same criterion is applied for water-silanol hydrogen bonds. The coordination numbers in Figures 3A-B (main text) take into account water-water and water-silanol HBs. The coordination number/water molecule is calculated at each time-step of the dynamics and then averaged over the whole trajectory. These coordination numbers have been calculated for each water molecule that belongs to the identified BIL (see section S5), and these coordination numbers have been plotted with respect to the average position of the water molecules along the trajectory, within the $x-y$ lateral directions of the silica surface, resulting in the $\mathrm{x}-\mathrm{y}$ spatial mapping shown in Figures 3A-B 
in the main text. This results in a 3D-map, where the color-coding (scale on the left-hand side of each figure) reports the average coordination number/water molecule. The scaling goes from dark blue zones for coordination numbers $<2.2$ to green-red zones for coordination numbers $\geq 3.1$. The reference value is 3.4 in bulk liquid water (obtained from AIMD simulations of bulk liquid water using the same set-up as the one employed here).

\section{S8 - Dependence of the MD results on the limited statistic of DFT-MD simulations.}

The rationalization, emerging from the DFT-MD results, for the way ions affect interfacial water structure and dynamics in the BIL is that both anion $\left(\mathrm{Cl}^{-}\right)$and cation $\left(\mathrm{K}^{+}\right)$are able to form inner sphere complexes with the silica surface when located in the BIL, hence completing their coordination shell using both BIL-water and surface SiOH (silanol) terminations. These ionsilanols interactions replace previously existing water-silanols H-Bonds, resulting in the formation of dangling $\mathrm{OH}$-groups which in turn reorient in-plane to form new water-water intra-BIL HBs, leading to the creation of an extended 2D-HB network (2DN) connecting BIL-water molecules via intra-BIL HBs (parallel to the surface). This highly connected 2D-HB-Network is reminiscent of the hydrophobic BIL-water revealed at the air-water interface ${ }^{29-30}$. This ultimately leads to a more homogeneous water coordination landscape above the surface and thus to a strong reduction of the number of under-coordinated water molecules in the BIL, which we propose to be the reason for the ion-induced acceleration of water vibrational relaxation observed at $\mathrm{pH}=2 \& 6$. One key point of this interpretation is that the ions $\left(\mathrm{K}^{+} \& \mathrm{Cl}^{-}\right)$form inner sphere complexes with the silica surface when located in the BIL.

Thus, we will first focus on ion configurations within the BIL. Since DFT-MD simulations do not provide enough sampling to correctly characterize the way ions are adsorbed at the silica-water 
interface, we show that our conclusion that $\mathrm{KCl}(\mathrm{NaCl})$ ions located in the $\mathrm{BIL}$ complete their hydration layer using both water and surface $\mathrm{SiOH}$ groups remains true also when randomly varying the ion position within the BIL in the total of $9 \mathrm{MD}$ simulations $(8$ with $\mathrm{KCl}+1$ with $\mathrm{NaCl}$, for a total of 150ps simulation time) that we have performed.

For each of the 9 simulations, the initial point has been chosen by randomly locating the ions in the simulation box, within a maximum initial distance of $6 \AA$ from the water surface. The ions are hence randomly located either in the BIL or in the first bulk water layer (as defined in section S5). Each simulation has been accumulated for $\sim 15-20$ ps after a short equilibration time, for a total of 150 ps.

Averaging over all the 8 simulations with $\mathrm{KCl}$, we first find that $\mathrm{K}^{+}$and $\mathrm{Cl}^{-}$spend the same total amount of time in the BIL, equal to $83 \%$ for $\mathrm{K}^{+}$and $81 \%$ for $\mathrm{Cl}^{-}$. For the remaining $17 \%$ / $19 \%$ of the time, both ions never reach distances larger than $8 \AA$ from the silica surface. This is in agreement with the DFT-MD simulation of the neat silica-water interface (see section S5) and with the experimental estimation of surface potential (see section S3), both showing that the system is at the isoelectric point, thus without an electrostatic driving force for the preferential adsorption of one ion over the other. The additional simulation with $\mathrm{NaCl}$ confirm this result, with both ions remaining for more than $85 \%$ of the time within the BIL.

The second and even more important result is that both $\mathrm{K}^{+} / \mathrm{Na}^{+}$and $\mathrm{Cl}^{-}$are found to systematically form inner sphere complexes when located in the BIL, independent of the kind of surface sites they interact with (i.e. germinal, isolated...), of the kind of ion pair they form (i.e. CIP, SSIP...), 
and of where they are located within the BIL. This shows that the conclusion obtained from DFTMD simulations presented in the main text and in sections S4 \& S5 are not an artifact of the limited statistic of DFT-MD and remains valid also when different ion configurations are explored.

In particular, the average (over all the trajectories) composition of the $\mathrm{K}^{+}$and $\mathrm{Cl}^{-}$coordination shells (when the ions are located in the BIL) is the following: 3.9 water +3.2 silanols for $\mathrm{K}^{+} \& 3.6$ water +2.3 silanols for $\mathrm{Cl}^{-}$. A similar result is obtained from the trajectory with $\mathrm{NaCl}$. See Table $\mathrm{S} 1$ for a summary. This shows that $\mathrm{NaCl}$ and $\mathrm{KCl}$ behave similarly. The total ions coordination resulting from ion-water + ion-silanol interactions is equivalent to the ion coordination in the bulk $^{25}$. Note also that these results are in agreement with the coordination shell composition calculated for $\mathrm{K}^{+}$and $\mathrm{Cl}^{-}$ions adsorbed at quartz-water interfaces from DFT-MD simulations in ref. $^{25}$. The trajectory of $20 \mathrm{ps}$ discussed in the main text corresponds to trajectory 1 in the following table.

\begin{tabular}{|l|l|l|l|l|l|}
\hline & $\mathrm{K}^{+}-\mathbf{H}_{2} \mathbf{O}$ & $\mathrm{K}^{+}-\mathrm{SiOH}$ & $\mathrm{Cl}^{-}-\mathbf{H}_{2} \mathbf{O}$ & $\mathrm{Cl}^{-}-\mathrm{SiOH}$ & Ion pair type \\
\hline Trajectory $\mathbf{1}$ & 4.2 & 2.0 & 3.1 & 3.0 & CIP \\
\hline Trajectory 2 & 3.6 & 4.3 & 4.8 & 2.0 & Far apart \\
\hline Trajectory 3 & 4.0 & 4.0 & 2.5 & 2.4 & CIP \\
\hline Trajectory 4 & 4.4 & 2.4 & 2.4 & 1.2 & CIP/SSIP \\
\hline Trajectory 5 & 3.9 & 4.2 & 4.0 & 2.2 & SSIP \\
\hline Trajectory 6 & 4.2 & 2.7 & 2.4 & 2.9 & CIP \\
\hline Trajectory 7 & 3.3 & 2.6 & 2.9 & 2.3 & CIP/SSIP \\
\hline Trajectory 8 & 5.0 & 3.5 & 4.8 & 1.6 & Far apart \\
\hline Average & $3.9 \pm 0.6$ & $3.2 \pm 0.8$ & $3.6 \pm 1.1$ & $2.3 \pm 0.6$ & / \\
\hline
\end{tabular}




\begin{tabular}{|l|l|l|l|l|l|}
\hline Traj. NaCl & 3.2 & 2.1 & 3.5 & 1.6 & CIP \\
\hline
\end{tabular}

Table S1. Coordination shell composition of $\mathrm{K}^{+} / \mathrm{Na}^{+}$and $\mathrm{Cl}^{-}$ions when located in the BIL. For each of the 9 independent simulations performed, the time-averaged $\mathrm{K}^{+} / \mathrm{Na}^{+}-\mathrm{SiOH}, \mathrm{K}^{+} / \mathrm{Na}^{+}$$\mathrm{H}_{2} \mathrm{O}, \mathrm{Cl}^{-}-\mathrm{SiOH}, \mathrm{Cl}^{-}-\mathrm{H}_{2} \mathrm{O}$ coordination numbers are reported, as well as the kind of configuration assumed by the ions during the simulation time, i.e. contact ion pair (CIP), solvent shared ion pair (SSIP, with part of the first hydration layer being shared in between the two ions) and far ions not forming an ion pair (far, with anion-cation distance larger than 6 $\AA$ ).

Now that preferential absorption of the $\mathrm{Cl}^{-} / \mathrm{Na}^{+} / \mathrm{K}^{+}$ions in inner-sphere mode has been proved for all trajectories, we present in Figure S10 the corresponding 9 plots for the time averaged spatial distribution of the coordination number of water in the BIL (like the ones shown in Figures 3A and $3 \mathrm{~B}$ in the main text). These plots allow us to evaluate the ions effect on the BIL-water HBstructure. 


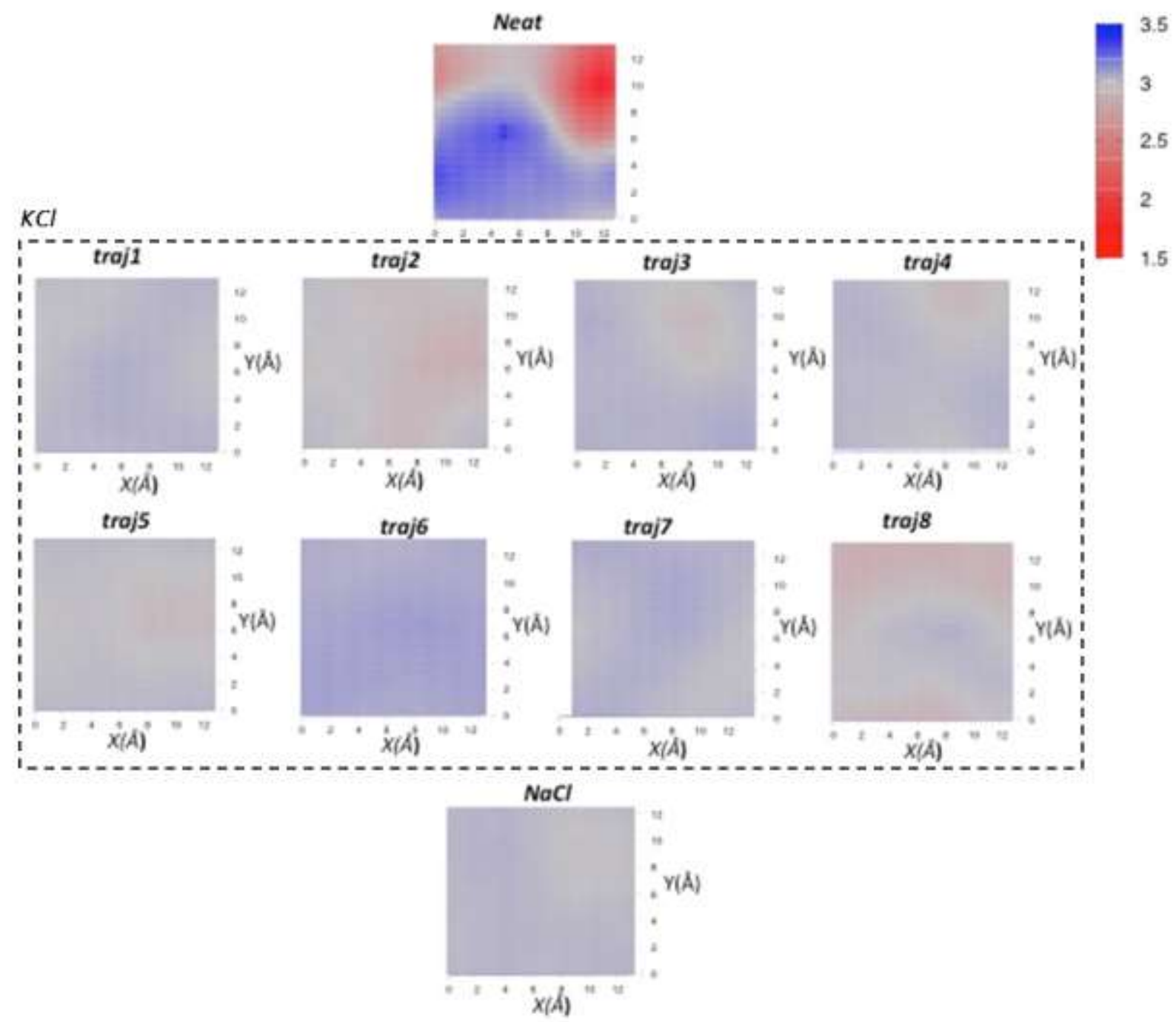

Figure S10. Time averaged coordination number of the water molecules in the BIL spatially resolved along the lateral $x$-y directions of the silica surface (in $\AA$ ) for: Neat aqueous silica interface (top), Each of the 8 trajectories (described in Table S1) with one $\mathrm{KCl}$ ion pair in the BIL (inside the dashed box) labeled as "traj1" to "traj8", Trajectory with one NaCl ion pair in the BIL (bottom). The color coding (vertical scale in the plots) goes from red (1.5 HBs/molecule) to blue $(3.5 \mathrm{HBs} /$ molecule). Note that "neat" and "traj1" are the same as the plots shown in the main text, but in a slightly modified scale.

Figure S10 confirms (for all 9 trajectories with ions) the conclusion reached in the main text, i.e. that $\mathrm{KCl} / \mathrm{NaCl}$ ions are able to order BIL-water molecules by inducing the formation of an airwater like 2D-HB-Network, resulting in a uniform 3-fold coordination for all water molecules in the BIL (vs the inhomogeneous and less connected BIL-water structure above the neat interface). This can be deduced from the homogeneous grey coloring in the plots for all the 9 trajectories with 
ions added to the interface vs the inhomogeneous coloring of the plot for the neat silica-water interface. In agreement, we also find that averaging over all the 9 simulations with one ion-pair in the BIL, a value of $1.74 \pm 0.09$ intra-BIL HBs/molecule (i.e. the in-plane oriented HBs forming the 2D-HB-Network) is obtained, coherent with the value of 1.7 intra-BIL HBs/molecule discussed in the main text.

\section{S9 - Estimation of BIL/DL contributions to TR-SFG based in the surface potential $\Delta \mathrm{V}$}

As discussed in the main text, the BIL/DL contributions to SS-SFG and TR-SFG (i.e. to the measured $T_{1}$ lifetimes) can be qualitatively estimated from the surface potential $(\Delta \mathrm{V})$, knowing that relaxation processes from both BIL/DL layers will contribute to the measured $\mathrm{T}_{1}$ lifetimes for a charged interface. This is because both BIL and DL layers are SFG active $\left(\left|\chi^{(2)}(\omega)\right|^{2}=\left|\chi^{(2)}{ }_{B I L}(\omega)+\chi^{(2)} \mathrm{DL}(\omega)\right|^{2}\right)$, with the DL-SFG signal being proportional to the surface potential drop across DL $\left(\Delta \phi_{\mathrm{DL}}\right)$ through $\left|\chi^{(2)}{ }_{\mathrm{DL}}(\omega)\right|^{2}=\left|\chi^{(3)}{ }_{\text {bulk }}(\omega)\right|^{2}\left(\Delta \phi_{\mathrm{DL}}\right)^{2}$. Note that $\Delta \phi_{\mathrm{DL}}$ defines the potential difference across the DL, which can, in first approximation, usually estimated as equal to the surface potential. However, for a quantitative estimation of the DL contribution, one should also consider the potential drop within the BIL layer, so that $\Delta \phi_{\mathrm{DL}}<\Delta \mathrm{V}$. The potential drop within the BIL is usually assumed negligible for large Debye lengths, i.e. for slow decaying surface potentials.

To better illustrate the deconvolution of the total SFG signals and $\mathrm{T}_{1}$ lifetimes into BIL/DL contributions, taking a theoretical point of view, we can consider that the total SFG (ppp) response of any aqueous interface is proportional to the Fourier transform of the sum of correlation functions between the $\mathrm{z}$-component of the dipole moment of each water molecule at time $\mathrm{t} 1\left(\mu_{\mathrm{z}, \mathrm{m}}(\mathrm{t} 1)\right)$ and 
the zz-element of the polarizability tensor of each water molecule at time t0 $\left(\alpha_{z z, l}(t 0)\right)$. For a system composed of $\mathrm{N}$ water molecules, the water SFG response will depend on $\mathrm{NxN}$ correlation terms (i.e. between all possible pairs of water molecules):

$$
\chi_{z z z}^{(2)}(\omega) \propto \sum_{\mathrm{m}=1}^{\mathrm{N}} \sum_{\mathrm{l}=1}^{\mathrm{N}} \int_{-\infty}^{+\infty} \mathrm{dt} \mathrm{e}^{\mathrm{i} \omega \mathrm{t}}\left\langle\mu_{\mathrm{z}, \mathrm{m}}(\mathrm{t} 1) \alpha_{\mathrm{zz}, \mathrm{l}}(\mathrm{t} 0)\right\rangle=\langle N N\rangle
$$

In the equation above, we named the sum of these $\mathrm{NxN}$ correlation terms as $\langle\mathrm{N} N\rangle$. By considering that both BIL/DL layers are SFG active for a charged interface, we can partition the sum over $m=1$ to $\mathrm{N}$ in the equation above into $\mathrm{N}_{\text {BIL }}+\mathrm{N}_{\text {DL }}$ where $\mathrm{N}_{\mathrm{BIL}}$ and $\mathrm{N}_{\text {DL }}$ are the numbers of water molecules within the BIL and DL regions, respectively. We hence obtain $\langle\mathrm{N} \mathrm{N}\rangle=\left\langle\mathrm{N}_{\mathrm{BIL}}\right.$ $\mathrm{N}\rangle+\left\langle\mathrm{N}_{D L} \mathrm{~N}\right\rangle$, where $\left\langle\mathrm{N}_{\mathrm{BIL}} \mathrm{N}\right\rangle$ and $\left\langle\mathrm{N}_{D L} \mathrm{~N}\right\rangle$ are respectively the $\mathrm{I}_{\mathrm{BIL}}$ and $\mathrm{I}_{\mathrm{DL}}$ discussed in the text, once integrated in the $\mathrm{OH}$ stretching region. By applying the same rationale to the second term in the correlation functions (i.e. to the sum over $\mathrm{l}=1$ to $\mathrm{N}$ in the equation above), we obtain that $\left\langle\mathrm{N}_{\mathrm{BIL}} \mathrm{N}\right\rangle=\left\langle\mathrm{N}_{\mathrm{BIL}} \mathrm{N}_{\mathrm{BIL}}\right\rangle+\left\langle\mathrm{N}_{\mathrm{BIL}} \mathrm{N}_{\mathrm{DL}}\right\rangle$ and $\left\langle\mathrm{N}_{\mathrm{DL}} \mathrm{N}\right\rangle=\left\langle\mathrm{N}_{\mathrm{DL}} \mathrm{N}_{\mathrm{BIL}}\right\rangle+\left\langle\mathrm{N}_{\mathrm{DL}} \mathrm{N}_{\mathrm{DL}}\right\rangle$, where $<$ $\mathrm{N}_{\mathrm{BIL}} \mathrm{N}_{\mathrm{DL}}>$ and $<\mathrm{N}_{\mathrm{DL}} \mathrm{N}_{\mathrm{BIL}}>$ are the BIL/DL cross-correlation terms.

The partition into $\mathrm{I}_{\mathrm{BIL}}$ and $\mathrm{I}_{\mathrm{DL}}$ hence takes into account BIL/DL cross correlation terms, in principle. However, in the present paper, we do not calculate theoretical vSFG spectra for pH 6 and 12 conditions, as the interpretation can be achieved with experiments and potential estimation alone.

To do this, we make use of the relationship $\mathrm{I}_{\mathrm{DL}} / \mathrm{I}_{\mathrm{BIL}} \alpha\left(\Delta \phi_{\mathrm{DL}}\right)^{2}$, i.e. the ratio of DL/BIL intensities is proportional to the square of the surface potential.

In order to be able to evaluate the ratio of DL/BIL intensities for any given $\Delta \phi_{\text {DL }}$ value, we need to know the value of $\mathrm{I}_{\mathrm{DL}} / \mathrm{I}_{\mathrm{BIL}}$ for a reference case, at a given $\Delta \phi_{\mathrm{DL}}$ value which will become our 
reference potential $\Delta \phi_{\mathrm{DL}}(\mathrm{ref})$. Once a reference is obtained, we can calculate $\mathrm{I}_{\mathrm{DL}} / \mathrm{I}_{\mathrm{BIL}}$ at any other $\Delta \phi_{\mathrm{DL}}$ value as:

$$
\mathrm{I}_{\mathrm{DL}} / \mathrm{I}_{\mathrm{BIL}}\left(\Delta \phi_{\mathrm{DL}}\right)=\mathrm{I}_{\mathrm{DL}} / \mathrm{I}_{\mathrm{BIL}}\left(\Delta \phi_{\mathrm{DL}}(\mathrm{ref})\right) *\left(\Delta \phi_{\mathrm{DL}} / \Delta \phi_{\mathrm{DL}}(\mathrm{ref})\right)^{2}
$$

According to our previous works on silica-water interfaces ${ }^{28,31}$, a value of $\mathrm{I}_{\mathrm{DL}} / \mathrm{I}_{\mathrm{BIL}} \sim 10$ is obtained for $\Delta \phi_{\mathrm{DL}} \sim 10 \mathrm{mV}$ (with both $\mathrm{I}_{\mathrm{DL}} / \mathrm{I}_{\mathrm{BIL}}$ and $\Delta \phi_{\mathrm{DL}}$ calculated ab initio from DFT-MD, see all details in ref. $\left.{ }^{28,31}\right)$. We hence take $\Delta \phi_{\mathrm{DL}}($ ref $)=10 \mathrm{mV}$ as the reference, with $\mathrm{I}_{\mathrm{DL}} / \mathrm{I}_{\mathrm{BIL}}\left(\Delta \phi_{\mathrm{DL}}(\mathrm{ref})\right)=10$.

Thus, Equation S7 is used to qualitatively estimate how much BIL and DL contribute to vSFG and hence to $\mathrm{T}_{1}$ relaxation time by making use of:

1) the theoretically calculated I-BIL/I-DL ratio for a silica-water interface with $\left(\Delta \phi_{D L}\right)=10 \mathrm{mV}$ (determined from ab-initio).

2) the relationship $\mathrm{I}_{\mathrm{DL}} / \mathrm{I}_{\mathrm{BIL}} \alpha\left(\Delta \phi_{\mathrm{DL}}\right)^{2}$, where we assume $\Delta \phi_{\mathrm{DL}}=\Delta \mathrm{V}$

The total measured $\mathrm{T}_{1}$ lifetime can be finally estimated as a first simple approximation by considering that the measured relaxation time can be physically interpreted as the weighted average of the relaxation processes involving different BIL/DL populations, so that: $\mathrm{T}_{1}=\mathrm{T}_{1}(\mathrm{BIL})$ $\mathrm{I}_{\mathrm{BIL}}+\mathrm{T}_{1}(\mathrm{DL}) \mathrm{I}_{\mathrm{DL}}$

Equation $\mathrm{S} 7$ leads e.g. to $\mathrm{I}_{\mathrm{DL}} / \mathrm{I}_{\mathrm{BIL}}\left(\Delta \phi_{\mathrm{DL}}\right)=2958$ for $\mathrm{pH} 12$ and no ions (where $\Delta \mathrm{V}=172 \mathrm{mV}$ ), and to $\mathrm{I}_{\mathrm{DL}} / \mathrm{I}_{\mathrm{BIL}}\left(\Delta \phi_{\mathrm{DL}}\right)=1464$ for $\mathrm{pH} 12+0.1 \mathrm{M}[\mathrm{NaCl}]($ where $\Delta \mathrm{V}=121 \mathrm{mV})$. See Table $\mathrm{S} 2$ below. 
The ratio is reduced if the $\Delta \phi_{\mathrm{DL}}$ is estimated with more accuracy by also accounting for the screening of the potential within the BIL region. In order to show this, we now do not consider anymore $\Delta \phi_{\mathrm{DL}}=\Delta \mathrm{V}$, but we take the potential values (from Figure S5) at a distance from the surface which correspond to the BIL/DL boundary.

From MD simulations, we know that the BIL/DL boundary is located at $0.3 \mathrm{~nm}$ from the instantaneous water surface (see section S6), which corresponds to a distance of 0.7-0.8 nm from the silica surface plane defined by the topmost heavy-atoms. Thus, if we take the $\Delta \mathrm{V}$ values at 1 $\mathrm{nm}$ from the solid surface, close to the BIL/DL boundary, we get $\Delta \phi_{\mathrm{DL}}=\sim 80 \mathrm{mV}$ for $\mathrm{pH} 12$ and no ions (instead of $\sim 200 \mathrm{mV}$ ) and $\Delta \phi_{\mathrm{DL}}=\sim 30 \mathrm{mV}$ for $\mathrm{pH} 12+$ ions (instead of $100 \mathrm{mV}$ ). These values, however, still lead to dominant DL contributions $\left(\mathrm{I}_{\mathrm{DL}} / \mathrm{I}_{\mathrm{BIL}}=690\right.$ and 80 , respectively; see Table S2), confirming the conclusion reached with the less accurate surface potential estimation.

We also notice that for $\mathrm{pH} 6$ and no ions, $\Delta \phi_{\mathrm{DL}}=80 \mathrm{mV}$ for both surface potential estimation methods (due to the slow decay typical of such a low ionic strength, i.e. $10^{-5}-10^{-7} \mathrm{M}$ ), while when ions are added to the system, the $\Delta \phi_{\text {DL }}$ value is very low $(\sim 4 \mathrm{mV})$ at $1 \mathrm{~nm}$ from the surface (Figure S5 \& Table S2), and so the DL-SFG contributions are negligible in the latter case.

\section{S10 - Interferences effect on the BIL/DL intensity ratio}

In our experiments, the ion concentration for the system with $\mathrm{pH} 6$ and $[\mathrm{NaCl}]=0 \mathrm{M}$ is expected to be on the order of $10^{-5}-10^{-7} \mathrm{M}$. For these concentration values the "effective" DL intensity which is experimentally measured at the silica-water interface (as well as any other charged aqueous interface), is known to be largely reduced due to destructive interferences ${ }^{27,32-34}$. For the silica- 
water interface investigated here, such behavior has been previously observed by Bonn et al. ${ }^{32}$ and Hore-Tyrode $^{34}$. From these works, it is evident that, depending on the experimental geometry, the DL intensity is decreased up to $20 \%$ of its original intensity (i.e. when interferences effect on the DL-SFG intensity are negligible).

In order to correct our estimated $\mathrm{I}_{\mathrm{DL}} / \mathrm{I}_{\mathrm{BIL}}$ ratio for interferences, following the derivation detailed in ref.34, we can recast Equation S7 in the form:

$$
\mathrm{I}_{\mathrm{DL}} / \mathrm{I}_{\mathrm{BIL}}\left(\Delta \phi_{\mathrm{DL}}\right)=\mathrm{I}_{\mathrm{DL}} / \mathrm{I}_{\mathrm{BIL}}\left(\Delta \phi_{\mathrm{ref}}=10 \mathrm{mV}\right) *\left|\Delta \phi_{\mathrm{DL}} \mathrm{f}_{3}\left(\kappa, \Delta \mathrm{k}_{\mathrm{z}}\right)\right|^{2} / \Delta \phi_{\mathrm{ref}}{ }^{2}
$$

Where $\mathrm{f}_{3}\left(\kappa, \Delta \mathrm{k}_{\mathrm{z}}\right)$ is the interference term, with $\mathrm{f}_{3}\left(\kappa, \Delta \mathrm{k}_{\mathrm{z}}\right)=\kappa /\left(\kappa-\mathrm{i} \Delta \mathrm{k}_{\mathrm{z}}\right), \kappa=$ inverse of the Debye Length and $\Delta \mathrm{k}_{\mathrm{z}}=$ wave vector mismatch for SFG photons generated at different probing depths ${ }^{34}$. The equation is here written by neglecting BIL-DL correlation terms for simplicity. This is possible because for such a large $\mathrm{I}_{\mathrm{DL}}$ value $\left(\mathrm{I}_{\mathrm{BIL}} / \mathrm{I}_{\mathrm{DL}}=640\right.$ before correcting for interferences, since $\Delta \phi_{\mathrm{DL}}=80 \mathrm{mV}$ ) including the cross terms would not alter the results and conclusions reached from the $\mathrm{I}_{\mathrm{BIL}} / \mathrm{I}_{\mathrm{DL}}$ estimation (i.e. that DL contributions are dominant for $\mathrm{pH} 6$ and no ions conditions). However, for a quantitative estimation of the BIL/DL contributions to the vSFG (which is out of the scope of the present investigation), the BIL/DL cross correlation terms should be explicitly included in the modeling.

By using Equation $\mathrm{S} 8$ to correct the $\mathrm{I}_{\mathrm{DL}} / \mathrm{I}_{\mathrm{BIL}}$ ratio for interferences, and knowing that at $\mathrm{pH} 6$ and no ions conditions $\Delta \phi_{\mathrm{DL}}=80 \mathrm{mV}$ (and $\left|\mathrm{f}_{3}\left(\kappa, \Delta \mathrm{k}_{\mathrm{z}}\right)\right|=0.2$ ), we obtain $\mathrm{I}_{\mathrm{DL}} / \mathrm{I}_{\mathrm{BIL}}=26$, i.e. the $\mathrm{DL}$ contribution is still dominant also after correcting for interferences. 


\begin{tabular}{|c|c|c|c|c|c|c|}
\hline & $\begin{array}{c}\Delta V(m V) \\
@ z=0 n m\end{array}$ & $\mathrm{I}_{\mathrm{DL}} / \mathrm{I}_{\mathrm{BIL}}$ & $\begin{array}{l}\Delta \phi_{\mathrm{DL}}(\mathrm{mV}) \\
@ \mathrm{z}=1 \mathrm{~nm}\end{array}$ & $I_{\mathrm{DL}} / \mathrm{I}_{\mathrm{BIL}}$ & $\begin{array}{c}\mathrm{I}_{\mathrm{DL}} / \mathrm{I}_{\mathrm{BIL}} \\
\text { (with Interference) }\end{array}$ & DL or BIL \\
\hline pH 12 & 172 & 2958.4 & 83 & 688.9 & 688.9 & $\mathrm{DL}$ \\
\hline $\mathrm{pH} 12+0.1 \mathrm{M} \mathrm{NaCl}$ & 121 & 1464.1 & 29 & 84.1 & 84.1 & $\mathrm{DL}$ \\
\hline $\mathrm{pH} 12+0.5 \mathrm{M} \mathrm{NaCl}$ & 90 & 810 & 7 & 4.9 & 4.9 & DL \\
\hline pH 6 & 81 & 656.1 & 81 & 656.1 & 26.2 & $\mathrm{DL}$ \\
\hline $\mathrm{pH} 6+0.1 \mathrm{M} \mathrm{NaCl}$ & 11 & 12.1 & 4 & 1.6 & 1.6 & $\mathrm{DL}+\mathrm{BIL}$ \\
\hline $\mathrm{pH} 6+0.5 \mathrm{M} \mathrm{NaCl}$ & 10 & 10 & 1 & 0.1 & 0.1 & $\mathrm{BIL}$ \\
\hline pH 2 & $6.0 \mathrm{E}-02$ & $3.6 \mathrm{E}-04$ & 4.4E-05 & 1.9E-10 & $1.9 \mathrm{E}-10$ & $\mathrm{BIL}$ \\
\hline $\mathrm{pH} 2+0.1 \mathrm{M} \mathrm{NaCl}$ & $3.0 \mathrm{E}-02$ & 9.0E-05 & $9.6 \mathrm{E}-06$ & $9.2 \mathrm{E}-12$ & $9.2 \mathrm{E}-12$ & $\mathrm{BIL}$ \\
\hline $\mathrm{pH} 2+0.5 \mathrm{M} \mathrm{NaCl}$ & 4.0E-03 & $1.6 \mathrm{E}-06$ & $4.2 \mathrm{E}-07$ & $1.8 \mathrm{E}-14$ & $1.8 \mathrm{E}-14$ & $\mathrm{BIL}$ \\
\hline
\end{tabular}

Table S2: IDL/I $\mathrm{IIL}$ ratio calculated for the surface potential at the silica surface $(\Delta \mathrm{V} @ \mathrm{z}=0$ $\mathrm{nm})$ and for the surface potential at the BIL-DL interface $\left(\Delta \phi_{\mathrm{DL}} @ \mathrm{z}=1 \mathrm{~nm}\right)$. Reduced $\mathrm{I}_{\mathrm{DL}} / \mathrm{I}_{\mathrm{BIL}}$ ratio is also calculated for the $\mathrm{pH} 6$ condition where the interference effect is expected, and the last column indicates whether the DL or the BIL dominates based on the $\mathrm{I}_{\mathrm{DL}} / \mathrm{I}_{\mathrm{BIL}}$ ratio.

\section{REFERENCES}

1. Tuladhar, A.; Piontek, S. M.; Borguet, E. Insights on Interfacial Structure, Dynamics, and Proton Transfer from Ultrafast Vibrational Sum Frequency Generation Spectroscopy of the Alumina (0001)/Water Interface. J. Phys. Chem. C 2017, 121, 5168-5177.

2. Eftekhari-Bafrooei, A.; Borguet, E. Effect of Surface Charge on the Vibrational Dynamics of Interfacial Water. J. Am. Chem. Soc. 2009, 131, 12034-12035.

3. Eftekhari-Bafrooei, A.; Borguet, E. Effect of Electric Fields on the Ultrafast Vibrational Relaxation of Water at a Charged Solid-Liquid Interface as Probed by Vibrational Sum Frequency Generation. J. Phys. Chem. Lett. 2011, 2, 1353-1358.

4. Tuladhar, A.; Dewan, S.; Kubicki, J. D.; Borguet, E. Spectroscopy and Ultrafast Vibrational Dynamics of Strongly Hydrogen Bonded OH Species at the $\alpha-\mathrm{Al}_{2} \mathrm{O}_{3}(1120) / \mathrm{H}_{2} \mathrm{O}$ Interface. J. Phys. Chem. C 2016, 120, 16153-16161.

5. Tuladhar, A.; Piontek, S. M.; Frazer, L.; Borguet, E. Effect of Halide Anions on the Structure and Dynamics of Water Next to an Alumina (0001) Surface. J. Phys. Chem. C 2018, $122,12819-12830$.

6. Dewan, S.; Yeganeh, M. S.; Borguet, E. Experimental Correlation Between Interfacial Water Structure and Mineral Reactivity. J. Phys. Chem. Lett. 2013, 4, 1977-1982. 
7. Eftekhari-Bafrooei, A.; Borguet, E. Effect of Hydrogen-Bond Strength on the Vibrational Relaxation of Interfacial Water. J. Am. Chem. Soc. 2010, 132, 3756-3761.

8. McGuire, J. A.; Shen, Y. R. Ultrafast Vibrational Dynamics at Water Interfaces. Science 2006, 313, 1945-1948.

9. $\quad$ Fecko, C. J.; Eaves, J. D.; Loparo, J. J.; Tokmakoff, A.; Geissler, P. L. Ultrafast Hydrogen-Bond Dynamics in the Infrared Spectroscopy of Water. Science 2003, 301, 16981702.

10. Lock, A. J.; Bakker, H. J. Temperature Dependence of Vibrational Relaxation in Liquid $\mathrm{H}_{2} \mathrm{O}$. J. Chem. Phys. 2002, 117, 1708-1713.

11. Cowan, M. L.; Bruner, B. D.; Huse, N.; Dwyer, J. R.; Chugh, B.; Nibbering, E. T. J.; Elsaesser, T.; Miller, R. J. D. Ultrafast Memory Loss and Energy Redistribution in the Hydrogen Bond Network of Liquid $\mathrm{H}_{2} \mathrm{O}$. Nature 2005, 434, 199-202.

12. Piontek, S. M.; Tuladhar, A.; Marshall, T.; Borguet, E. Monovalent and Divalent Cations at the alpha- $\mathrm{Al}_{2} \mathrm{O}_{3}(0001) /$ Water Interface: How Cation Identity Affects Interfacial Ordering and Vibrational Dynamics. J. Phys. Chem. C 2019, 123, 18315-18324.

13. Lock, A. J.; Woutersen, S.; Bakker, H. J. Ultrafast Energy Equilibration in HydrogenBonded Liquids. J. Phys. Chem. A 2001, 105, 1238-1243.

14. Rey, R.; Møller, K. B.; Hynes, J. T. Ultrafast Vibrational Population Dynamics of Water and Related Systems: A Theoretical Perspective. Chem. Rev. 2004, 104, 1915-1928.

15. Dove, P. M. The Dissolution Kinetics of Quartz in Sodium Chloride Solutions at 25 Degrees to 300 Degrees C. Am. J. Sci. 1994, 294, 665-712.

16. Bard, A. J.; Faulkner, L. R. Fundamentals and Applications. Electrochemical Methods 2001, $2,482$.

17. Bard, A. J.; Faulkner, L. R.; Leddy, J.; Zoski, C. G., Electrochemical Methods: Fundamentals and Applications; Vol. 2, Wiley: New York, 1980.

18. Ong, S.; Zhao, X.; Eisenthal, K. B. Polarization of Water Molecules at a Charged Interface: Second Harmonic Studies of the Silica/Water Interface. Chem. Phys. Lett. 1992, 191, 327-335.

19. Ugliengo, P.; Sodupe, M.; Musso, F.; Bush, I. J.; Orlando, R.; Dovesi, R. Realistic Models of Hydroxylated Amorphous Silica Surfaces and MCM-41 Mesoporous Material Simulated by Large-scale Periodic B3LYP Calculations. Advanced Materials 2008, 20, 45794583.

20. VandeVondele, J.; Krack, M.; Mohamed, F.; Parrinello, M.; Chassaing, T.; Hutter, J. Quickstep: Fast and Accurate Density Functional Calculations Using a Mixed Gaussian and Plane Waves Approach. Computer Physics Communications 2005, 167, 103-128.

21. Becke, A. D. Density-Functional Exchange-Energy Approximation with Correct Asymptotic Behavior. Phys. Rev. A 1988, 38, 3098-3100.

22. Lee, C.; Yang, W.; Parr, R. G. Development of the Colle-Salvetti Correlation-Energy Formula into a Functional of the Electron Density. Phys. Rev. B 1988, 37, 785-789.

23. Stefan, G. Semiempirical GGA-type Density Functional Constructed with a Long-Range Dispersion Correction. J. Comput. Chem. 2006, 27, 1787-1799.

24. Goedecker, S.; Teter, M.; Hutter, J. Separable Dual-Space Gaussian Pseudopotentials. Phys. Rev. B 1996, 54, 1703-1710.

25. Pfeiffer-Laplaud, M.; Gaigeot, M.-P. Adsorption of Singly Charged Ions at the Hydroxylated (0001) $\alpha$-Quartz/Water Interface. J. Phys. Chem. C 2016, 120, 4866-4880. 
26. Pfeiffer-Laplaud, M.; Gaigeot, M.-P. Electrolytes at the Hydroxylated (0001) $\alpha$ Quartz/Water Interface: Location and Structural Effects on Interfacial Silanols by DFT-Based MD. J. Phys. Chem. C 2016, 120, 14034-14047.

27. Wen, Y.-C.; Zha, S.; Liu, X.; Yang, S.; Guo, P.; Shi, G.; Fang, H.; Shen, Y. R.; Tian, C. Unveiling Microscopic Structures of Charged Water Interfaces by Surface-Specific Vibrational Spectroscopy. Phys. Rev. Lett. 2016, 116, 016101.

28. Pezzotti, S.; Galimberti, D. R.; Shen, Y. R.; Gaigeot, M.-P. Structural Definition of the BIL and DL: a New Universal Methodology to Rationalize Non-Linear $\chi^{(2)}(\omega)$ SFG Signals at Charged Interfaces, Including $\chi^{(3)}(\omega)$ Contributions. Phys. Chem. Chem. Phys. 2018, 20, 51905199.

29. Pezzotti, S.; Galimberti, D. R.; Gaigeot, M.-P. 2D H-Bond Network as the Topmost Skin to the Air-Water Interface. J. Phys. Chem. Lett. 2017, 8, 3133-3141.

30. Pezzotti, S.; Serva, A.; Gaigeot, M.-P. 2D-HB-Network at the Air-Water Interface: A Structural and Dynamical Characterization by Means of Ab Initio and Classical Molecular Dynamics Simulations. J. Chem. Phys. 2018, 148, 174701.

31. Pezzotti, S.; Galimberti, D.; Shen, Y.; Gaigeot, M.-P. What the Diffuse Layer (DL) Reveals in Non-Linear SFG Spectroscopy. Minerals 2018, 8, 305.

32. Schaefer, J.; Gonella, G.; Bonn, M.; Backus, E. H. G. Surface-Specific Vibrational Spectroscopy of the Water/Silica Interface: Screening and Interference. Phys. Chem. Chem. Phys. 2017, 19, 16875-16880.

33. Gonella, G.; Lütgebaucks, C.; de Beer, A. G. F.; Roke, S. Second Harmonic and SumFrequency Generation from Aqueous Interfaces Is Modulated by Interference. J. Phys. Chem. C 2016, 120, 9165-9173.

34. Hore, D. K.; Tyrode, E. Probing Charged Aqueous Interfaces Near Critical Angles: Effect of Varying Coherence Length. J. Phys. Chem. C 2019, 123, 16911-16920. 